Seminal fluid enhances sperm viability in the leafcutter ant Atta colombica

Den Boer, Susanne Petronella A; Boomsma, Jacobus Jan; Baer, Boris

Published in:

Behavioral Ecology and Sociobiology

DOI:

$10.1007 / \mathrm{s} 00265-008-0613-5$

Publication date:

2008

Document version

Publisher's PDF, also known as Version of record

Citation for published version (APA):

Den Boer, S. P. A., Boomsma, J. J., \& Baer, B. (2008). Seminal fluid enhances sperm viability in the leafcutter ant Atta colombica. Behavioral Ecology and Sociobiology, 62(12), 1843-1849. https://doi.org/10.1007/s00265008-0613-5 


\title{
Seminal fluid enhances sperm viability in the leafcutter ant Atta colombica
}

\author{
Susanne P. A. den Boer • Jacobus J. Boomsma • \\ Boris Baer
}

Received: 25 April 2008/Revised: 28 May 2008 / Accepted: 3 June 2008/Published online: 3 July 2008

(C) Springer-Verlag 2008

\begin{abstract}
The seminal fluid that accompanies sperm in ejaculates has been shown or suggested to affect sperm competition and paternity success of insects by preventing female remating, inducing oviposition, and forming mating plugs. In Atta leafcutter ants, queens have multiple mates but never remate later in life, although they may live and produce fertilized eggs for several decades. The mating biology and life history of these ants therefore suggests that the major function of seminal fluid is to maximize sperm viability during copulation, sperm transfer, and initial sperm storage. We tested this hypothesis by comparing the viability of testis sperm and ejaculated sperm (mixed with seminal fluid) and found a significant positive effect of seminal fluid on sperm viability. We further quantified this positive effect by adding accessory gland secretion (a major component of seminal fluid) in a dilution series, to show that minute quantities of accessory gland secretion achieve significant increases in sperm viability. Sperm stored by queens for 1 year benefited in a similar way from being
\end{abstract}

Responsible editor: J. Heinze

S. P. A. den Boer $(\varangle) \cdot J$. J. Boomsma $\cdot$ B. Baer

Centre for Social Evolution, Department of Biology,

University of Copenhagen,

Universitetsparken 15,

2100 Copenhagen, Denmark

e-mail: spadenboer@bio.ku.dk

B. Baer

ARC Centre of Excellence in Plant Energy Biology, MCS

Building M316, The University of Western Australia,

Crawley 6009, Australia

B. Baer

Centre for Evolutionary Biology, School of Animal Biology

(M092), The University of Western Australia,

Crawley 6009, Australia exposed to accessory gland compounds after dissection in control saline solution. Our results provide the first empirical evidence that seminal fluid is important for the production of viable ejaculates and that the accessory glands of Atta males - despite their small size-are functional and produce a very potent secretion.

Keywords Social insects · Mating · Sperm storage $\cdot$ Sperm transfer $\cdot$ Spermatheca $\cdot$ Accessory glands

\section{Introduction}

During copulation, insect males do not only transfer sperm but also seminal fluid to the female, which consists of several secretions that are mostly produced by the male accessory glands (Chapman and Davies 2004). More than 100 accessory gland proteins have so far been identified in the fruit fly Drosophila melanogaster (Ravi Ram and Wolfner 2007) and some of these have been experimentally shown to reduce female receptivity and longevity or to enhance egg laying (the "sex peptides"-Kubli 2003). These proteins manipulate female physiology as they seem able to enter the hemolymph and bind to specific receptor sites in the nervous system (Yapici et al. 2008). Manipulative compounds in the seminal fluid are generally considered to be involved in sperm competition and in mediating arms races between the sexes in insects with promiscuous mating systems (reviewed by Chapman and Davies 2004; Chen 1984; Gillott 2003; Poiani 2006; Ravi Ram and Wolfner 2007; Simmons 2001). Several experiments have shown that seminal fluid also influences ejaculate quality characteristics such as sperm motility and fertilization capacity (reviewed by Poiani 2006). However, even though it has been suggested (Poiani 2006; Thomas 
and Simmons 2007), no experiments showing an effect of seminal fluid on sperm viability have been conducted.

Seminal fluid is specifically interesting to study in eusocial insects (termites, ants, some bees, and wasps) because they have "pair bonding" for life (admittedly as stored sperm in the eusocial Hymenoptera). Throughout these groups, all mate choice decisions are made early in a queen's life and remating promiscuity is absent once queens have started to lay eggs (Boomsma 2007). Ant males normally have a fixed supply of sperm when they eclose (Hölldobler and Bartz 1985), which implies that they can obtain only a fixed number of (usually few) matings during a single swarming event after which they die (Boomsma et al. 2005). Males are thus greatly survived by their sperm within the sperm storage organ (spermatheca) of queens, which may have a maximum lifespan of several decades (Keller 1998) and produce millions of offspring. It is therefore crucial for males to produce highly viable ejaculates that stay alive during mating, sperm transfer, and initial sperm storage and for queens to further enhance sperm survival during the years of storage that follow. Selection for male traits that promote short-term sperm viability and queen traits that promote long-term sperm viability must therefore have been strong.

The long life of ant queens and the absence of remating promiscuity led us to hypothesize that the seminal fluid of ants is important for maintaining high sperm viability. Even in the derived lineages of ants where queens mate with multiple males during a single mating event, selection for compounds that increase individual male paternity at the expense of female fitness should be weak after sperm storage. There is a long time lag between insemination and sexual reproduction (years in the species investigated here) and queens have to produce multiple broods of sterile workers before their colonies are large enough to produce sexual offspring (Baer 2003; Baer and Boomsma 2004). Any seminal fluid trait that would compromise queen survival or long-term fertility would thus have a negative effect on the fitness of all males represented in a queen's spermatheca, as weaker colonies are unlikely to survive until first reproduction. However, to our knowledge, the effect of seminal fluid on sperm viability in social insects has only been inferred (Collins et al. 2006; Baer et al. unpublished data) but never been empirically tested. The few available studies of seminal fluid in the eusocial Hymenoptera have focused on the mating plug function of accessory gland secretion in bees and fire ants (Baer et al. 2001; Collins et al. 2006; Colonello and Hartfelder 2005; Mikheyev 2003; Sauter et al. 2001).

Here, we provide a direct test of the hypothesized positive effect of seminal fluid, and its major component accessory gland secretion, on sperm viability in the leafcutter ant Atta colombica. The queens of this species typically mate with two to three males (Baer and Boomsma 2006; Fjerdingstad et al. 1998) and, unlike most other eusocial insects, sperm is directly transferred to the spermatheca (Baer and Boomsma 2006). Any interejaculate competition prior to sperm storage as found in promiscuous nonsocial insect species is therefore absent. Competition between the clonal ejaculates of haploid males within the spermatheca might exist, but queens should have been under intense selection to control sperm death because they cannot remate later in life and the total amount of stored sperm is likely to determine their lifetime fitness (Cole 1983). Over evolutionary time, Atta leafcutter ants have taken queen fecundity and long-term storage of sperm to extremes. A. colombica queens are known to initially store up to 450 million sperm and to use them economically enough to sire millions of offspring over several decades (Baer et al. 2006; Fjerdingstad and Boomsma 1998; Weber 1972). This species is therefore particularly suitable to test the effect of seminal fluid on sperm viability.

\section{Material and methods}

All ants were collected in Gamboa, Republic of Panama, in May 2006 and May 2007. Males were sampled from several mature colonies and queens were collected by either excavating 1-year-old colonies or collecting inseminated founding queens in their incipient nest burrows a few days after the mating flight. One-year-old colonies can be easily recognized because they typically contain one (rarely two) fungus chambers and their nests have only a single entrance funnel. Dissections were carried out with Inox 5 (Biology) watchmaker forceps in Hayes solution $(9 \mathrm{~g} \mathrm{NaCl}, 0.2 \mathrm{~g}$ $\mathrm{CaCl}_{2}, 0.2 \mathrm{~g} \mathrm{KCl}$, and $0.1 \mathrm{~g} \mathrm{NaHCO}_{3}$ in $1,000 \mathrm{ml} \mathrm{H}_{2} \mathrm{O}$ ). Hayes solution was chosen both for dissections and as a sperm diluent because it is a relatively simple saline solution that would not have complex biochemical interactions with either the seminal fluid or the sperm. Because Hayes was originally developed as a semen extender for honeybees (Schley 1987), we assumed that it represented an environment that is physically similar to the ejaculate but lacks proteins and metabolites such as carbohydrates, fatty acids, or amino acids. Hayes would thus be expected to impose some physiological stress on sperm, resulting in increasing sperm mortality over time, an expectation that we confirmed in a set of pilot experiments. This made Hayes ideal as a control solution in order to quantify the positive effects of seminal fluid on sperm survival in a suitable but suboptimal environment. Exposure to Hayes saline and the handling of sperm itself (pipetting and exposing it to air) will thus negatively affect sperm viability (Damiens et al. 2002), whereas adding seminal fluid was hypothesized to counter this effect. Our experiments 
therefore measured the relative sperm-enhancing effects of seminal fluid in a standardized and carefully controlled way but not the absolute sperm viabilities as one would do when optimizing an artificial insemination technique.

Sperm viability was measured using the Live/Dead ${ }^{\mathrm{TM}}$ sperm viability kit (L-7011, Molecular Probes), which consists of a membrane-permeant nucleic acid stain for live sperm (SYBR-14) and a dead cell stain (propidium iodide). For each measurement, $5 \mu$ SYBR-14 working solution $(2 \mu \mathrm{l}$ SYBR-14 stock in $98 \mu$ l Hayes saline) was added to $5 \mu \mathrm{l}$ of the sperm samples and incubated for $10 \mathrm{~min}$ on a microscope slide. Afterwards, $2 \mu \mathrm{l}$ of propidium iodide was added to each sample and incubated for $7 \mathrm{~min}$ at room temperature in a dark humid box to prevent desiccation. Sample preparation time prior to sperm viability counts typically took around $30 \mathrm{~min}$ and did not differ substantially between samples and treatments. The number of live, dead, and dual-stained (both green and red) sperm was then counted for a minimum of 400 randomly selected sperm cells per sample, using a fluorescence microscope (Olympus CX41, EXFO X-Cite 120, filter cube CX-DMB-2, $\times 400-800$ magnification). Dual-stained cells (max $1.5 \%$ per sample) were regarded as dying cells (Damiens et al. 2002) and included in our live cell counts since they were assumed to have been alive when the sample was taken. Sperm viability was calculated for each sample as the percentage of live sperm in the total number of sperm counted.

To validate the experimental protocol, sperm were killed by either freezing for $24 \mathrm{~h}$ at $-20^{\circ} \mathrm{C}$ or autoclaving for $20 \mathrm{~min}$ at $121^{\circ} \mathrm{C}$. As expected all sperm in these samples were stained red (dead).

To test for the effect of the seminal fluid on sperm viability, we dissected 17 sexually mature males sampled from two different colonies and compared sperm viability between ejaculated sperm and sperm collected directly from the male sperm storage organs, the accessory testes (Fig. 1). To obtain ejaculated sperm, we dissected one accessory testis and gently squeezed it with soft forceps. This resulted in a contraction of the accessory testis, causing sperm to be forcefully expelled through the ejaculatory duct while passing the accessory glands where secretion is added. A $2 \mu \mathrm{l}$ sample of ejaculate was collected with a pipette. For each male, we also obtained a sperm sample from the accessory testis, by puncturing the second accessory testis and collecting $2 \mu \mathrm{l}$ of outflowing sperm. As no difference in sperm viability between left and right accessory testis was found ( $t$ test, $t=0.919, p=0.326, n=26$ ), sampling was randomized between left and right accessory testes. All sperm samples were diluted in $1 \mathrm{ml}$ Hayes and a $5 \mu \mathrm{l}$ subsample was used to measure sperm viability.

Male accessory gland secretions make up almost all of the seminal fluid. We tested for the effects of these

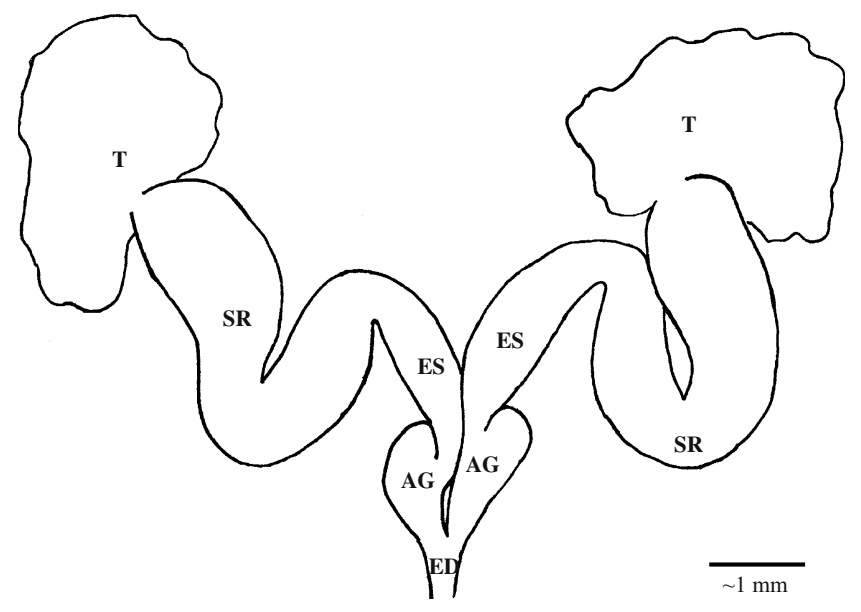

Fig. 1 Schematic drawing of the sexual organs of a mature $A$. colombica male. The testes $(T)$ degenerate after eclosion as adult. Sperm is stored in the sperm reservoirs $(S R)$, which together with the ejaculate sections $(E S)$ form the accessory testes $(A T)$. During ejaculation, sperm is forcefully expelled from the ES through the ejaculatory duct $(E D)$ passing the accessory glands $(A G)$ so that accessory gland compounds are added to sperm during ejaculation

secretions on the viability of sperm sampled from male testes and queen spermathecae. To do this, we excavated 240 males from a single colony. Their accessory gland pairs were dissected and added to a vial with $24 \mathrm{ml}$ Hayes, ruptured with watchmaker forceps shortly after collection, and vortexed for $1 \mathrm{~min}$. We then centrifuged the sample at $10,000 \mathrm{rpm}$ for $4 \mathrm{~min}$ to separate the soluble gland secretions (supernatant) from the gland tissue (pellet). The supernatant was used to create a dilution series that finally contained the secretion equivalents of zero, one, two, four, ten, and 20 accessory glands per milliliter Hayes saline. We then collected six sperm samples $(2 \mu$ leach) from the accessory testes of six brother males and from the spermathecae of six newly inseminated queens. The sperm samples were gently dissolved in the different accessory gland dilutions and a $5 \mu \mathrm{l}$ sample of each of these sperm solutions was used to determine sperm viability.

To test whether the effect of accessory gland compounds differs between short- and long-term stored sperm, we obtained a second dilution series of accessory gland secretion, using another sample of 240 males. We also excavated six newly inseminated queens and six queens from 1-year-old colonies (see methods described above). We collected sperm samples from the spermathecae of these queens and dissolved them in six different accessory gland dilutions, before determining sperm viability.

To estimate the dilution factor of accessory gland secretion in our experimental series, we estimated the volume of male accessory glands from digital photos of one gland in ten males. Both the area and the maximal length were measured, using the program Image $\mathrm{J} 1.38 \times$ (freely available at http://rsb.info.nih.gov/ij/). As the glands 
are cylindrical in shape, their volumes could be estimated, producing a mean accessory gland volume of $1.286 \pm$ $0.135 \mathrm{~mm}^{3}$ (mean $\pm \mathrm{SEM}$ ). This figure was used to convert concentrations expressed as number of glands per milliliter Hayes into approximate dilution factors (Fig. 3).

Statistical analyses were carried out using JMP 5.1 and SPSS 13.0 for Windows. Repeated-measures analysis of variance (ANOVA) was used to test for differences in sperm viability between testes and ejaculates in the first experiment, using sampling origin (accessory testis or ejaculate) as within-subject factor and colony as betweensubject factor. Analysis of covariance (ANCOVA) was used to test for the effect of accessory gland concentration (dilution factor) on sperm viability in the second experiment. Data were linearized with a reciprocal transformation $(1 /(x+1))$, where $x$ is the accessory gland concentration. The type of individuals tested (male versus queen and newly inseminated queen versus 1-year-old queen) was treated as main effect and the dilution factor as covariate.

\section{Results}

As expected, dissolving sperm in Hayes saline led to relatively low sperm viability values (Fig. 2). However, significantly higher proportions of live sperm were found in the samples of artificially induced ejaculates that contained

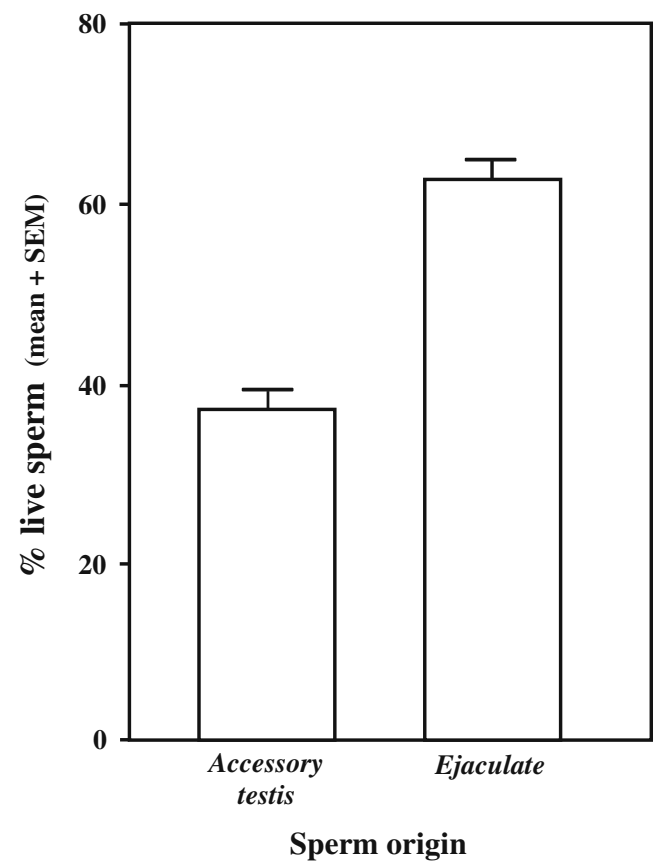

Fig. 2 Sperm viability in samples taken from the accessory testes or from ejaculates. The mean percentage of live sperm $( \pm \mathrm{SEM})$ for samples from the accessory testes was $36.95 \pm 1.98 \%$ and for samples from the ejaculate $62.54 \pm 2.12 \%, n_{\text {total }}=17$

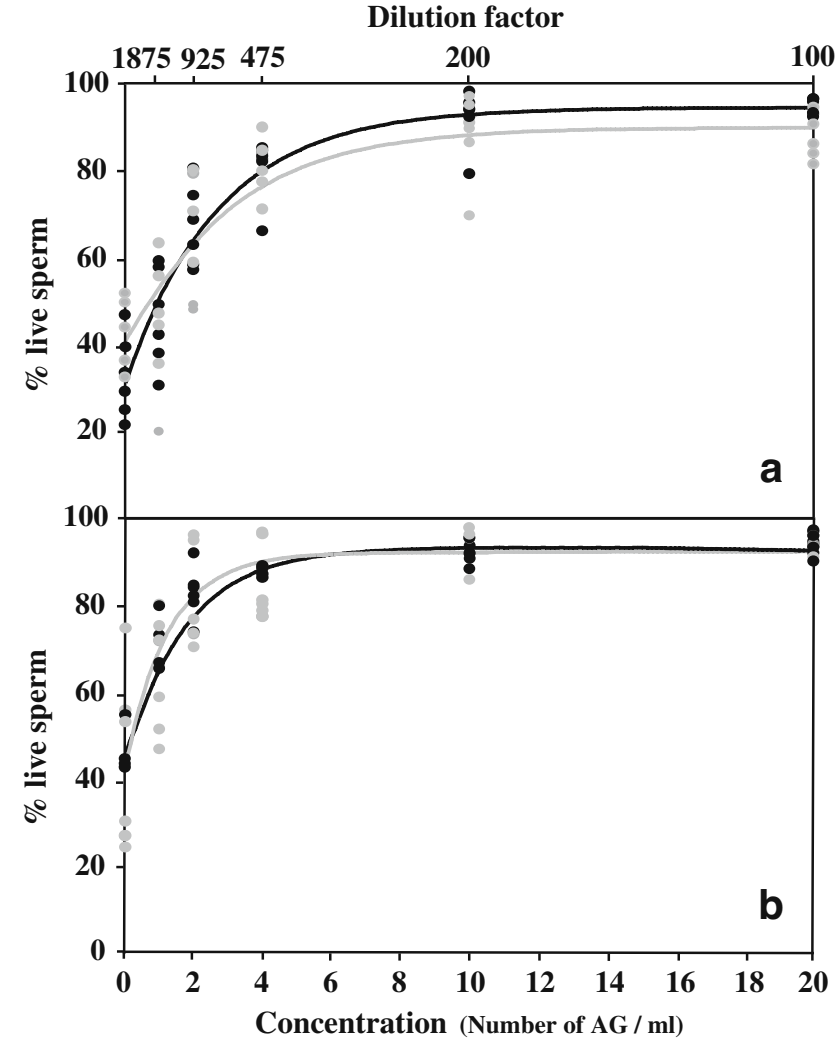

Fig. 3 The effect of increasing concentrations of accessory gland secretion on the viability of $A$. colombica sperm. a Sperm taken from the accessory testes of males (black dots and line) compared to sperm taken from the spermathecae of newly inseminated queens (gray dots and line). $\mathbf{b}$ Sperm taken from the spermathecae of newly inseminated queens (gray dots and line) and 1-year-old queens (black dots and line). The estimated approximate dilution factors of the accessory gland secretions are given above the panels. When no accessory gland secretion is added, sperm viabilities (mean \pm SEM) for a were $32.51 \pm 3.85 \%$ (males) and $41.26 \pm 3.43 \%$ (founding queens) and for b $42.63 \pm 3.64 \%$ (founding queens) and $44.25 \pm 8.24 \%$ (1-year-old queens). When the secretion of 20 accessory glands was added (approximately 100 times more diluted than secretion in situ) sperm viabilities (mean \pm SEM) for a were $94.71 \pm$ $0.78 \%$ (males) and $88.42 \pm 2.14 \%$ (founding queens) and for b $94.07 \pm$ $1.79 \%$ (founding queens) and $94.12 \pm 2.82 \%$ (1-year-old queens). Curve fitting and statistics were done on transformed $(1 /(1+x))$ dilution data, which linearized the relationships

seminal fluid compared to the sperm-only samples taken directly from the accessory testes of sexually mature males. This difference was consistent across males from different colonies (repeated measures ANOVA: sample origin $F_{1,15}=$ 77.532, $p<0.001$, colony $F_{1,15}=1.013, p=0.330$, Fig. 2). This implies that seminal fluid components enhance sperm survival and that the effect is strong because the sperm samples were diluted about 900 times prior to the sperm viability measurements.

Sperm viability increased sharply with increasing concentrations of accessory gland secretion, both for sperm taken from male accessory testes and from queen spermathecae (ANCOVA: accessory gland concentration, $F_{1,68}=$ 
295.17, $p<0.001$; sample origin $F_{1,68}=0.15, p=0.698$; Fig. 3). The curve for spermatheca sperm was slightly shallower than the curve for testes sperm, but this difference was not significant (accessory gland concentration $\times$ sample origin $F_{1,68}=2.96, p=0.0899$ ).

Similar effects were found when comparing the effect of seminal fluid on sperm that had been stored for shorter (a few days) or longer (approximately 12 months) periods of time (ANCOVA: accessory gland concentration, $F_{1,68}=239.254$, $p<0.001$; queen age, $F_{1,68}=0.32, p=0.859$; Fig. 3a). Here, the two curves were completely parallel (queen age $\times$ accessory gland concentration, $F_{1,68}=0.23, p=0.879$ ). This indicates that the viability-enhancing effect of seminal fluid after exposing sperm to the stressful Hayes conditions is independent of sperm age, which suggests that sperm viability after a year of storage is as high as directly after insemination.

\section{Discussion}

Our present study provides the first evidence that seminal fluid, and its major component accessory gland secretion, is of significant importance for sperm viability, even at very low concentrations. Even when the accessory gland secretion of a single male (two accessory glands) was about 900 times diluted (Fig. 3), twice the number of sperm cells survived compared to the control treatment where no accessory gland secretion was added to the Hayes saline solution ( $y$-intercepts in Fig. 3a). The potent effects of accessory gland secretion is also apparent from the 100 times dilutions (ten glands in $1 \mathrm{ml}$ ), which increased sperm viability to approximately $90 \%$, i.e., very close to the maximum viabilities that we were able to measure. It is important to note that the above-mentioned volume estimates of accessory gland secretion that an average male contributes to an ejaculate might be overestimations because they were based on the volume of the entire gland rather than the secretory lumen only. Furthermore, most males will not use all their seminal fluid for a single ejaculate, as they are likely to mate with several queens (Baer and Boomsma 2004). Our estimates of the positive effect of seminal fluid on sperm viability are therefore conservative.

Earlier work has shown that the accessory glands in Atta are small compared to the less derived genera within the fungus-growing ants that have maintained single mating of queens (Baer and Boomsma 2004). The relative size of the testes showed an opposite trend, which suggests that males in the multiple-mated species of Atta and Acromyrmex have been selected to increase the number of sperm per ejaculate at the expense of the accessory gland compounds responsible for mating plugs in the singly mated species. However, our results now show that although the glands are small (Fig. 1), they are very active and produce secretions that have a strong effect on sperm viability. Thus, although the accessory glands might have lost some of their original functions, selection on males appears to have enhanced other functions, which are vital for the production of the large high-quality ejaculates that are needed when sperm is to be stored for decades.

It has recently been hypothesized that males may benefit from the presence of another male's seminal fluid in the female reproductive tract (Hodgson and Hosken 2006), so that they can reduce their seminal fluid contribution per ejaculate and smaller accessory glands could become selected over time. We note, however, that this would assume that accessory gland secretion is as beneficial to own sperm as to alien sperm and that this effect would be limited in Atta leafcutter ants that mate with two to three males, relative to promiscuous nonsocial insects mating with many males.

Direct sperm storage without competition between ejaculates

One of the reasons why we expected high sperm viability is that sperm of A. colombica is transferred directly to the spermatheca (Baer and Boomsma 2006). Similar forms of direct sperm transfer to the spermatheca have so far only been found in the dwarf honeybees Apis florea and Apis adreniformis (Koeniger and Koeniger 1991; Koeniger et al. 1989). In most other eusocial insects, sperm seems to be deposited in the bursa copulatrix and it may take hours or even days before the spermatheca is completely filled and the remaining sperm is discarded (Woyke 1983). In the honeybee for example, sperm is transferred to the spermatheca over a time period of $90 \mathrm{~h}$, but only $3-5 \%$ of all sperm acquired during a nuptial flight becomes stored (Baer 2005). Such a sperm storage process offers the possibility for hostile seminal fluid traits to evolve as long as they will guarantee that sufficient numbers of live sperm are ultimately stored and queen fitness is either not affected or benefits from storing the most viable sperm. In contrast, direct sperm transfer as found in Atta prevents viability-based competition between individual sperm for storage, even though some sperm competition might still take place within the spermatheca (Boomsma et al. 2005). This underlines that, in species where sperm is ejaculated directly into the spermatheca, the seminal fluid components are likely to be selected primarily for their sperm-viability-enhancing function, rather than as weapons of sexual conflict.

Nothing is known about sperm viability in the dwarf honeybees, but stored sperm of the domestic honeybee Apis mellifera has similarly high viability as we estimated for stored A. colombica sperm (see Fig. 3; Collins 2000; Collins and Donoghue 1999; Hunter and Birkhead 2002). 
This suggests that similar selection forces for high sperm viability apply, but it remains to be seen whether accessory gland secretions of honeybees have convergently evolved the same viability-enhancing functions as in Atta. Queens of both leafcutter ants and honeybees should have been under selection to reduce or eliminate any interejaculate sperm competition after storage since the consequences of reduced overall sperm viability at this stage will almost certainly reduce the lifetime fitness of queens. Viabilitybased competition between ejaculates in the female reproductive tract has so far only been found in the nonsocial stalk-eyed fly Cyrtodiopsis whitei, where it has been shown that the seminal fluid of a normal male negatively affects sperm viability of meiotic drive males (Fry and Wilkinson 2004). It would also be interesting to examine the potential effects of "self" and "nonself" seminal fluid on sperm viability in leafcutter ants and honeybees.

The effect of seminal fluid on sperm survival did not differ between sperm taken from the accessory testes or queen's spermatheca (Fig. 3a). This suggests that the viability-enhancing effect of seminal fluid compounds does not have a lasting effect because spermatheca sperm would otherwise have been more robust (i.e., have expressed higher viability after being dissected in Hayes). When sperm suffers from stress factors such as exposure to air and Hayes solution, it apparently benefits from seminal fluid compounds independent of whether it has been exposed to the seminal fluid a few days or an entire year earlier. Also, when comparing the viability of ejaculated sperm (Fig. 2) with the average viability of spermathecal sperm of the same dilution $(A G=2$; Fig. 3a), no differences in viability were observed. Female-mediated sperm death as hypothesized for other insects (e.g., Bernasconi et al. 2002; Demary 2005) therefore does not seem to occur in A. colombica. This is not surprising since a queen's reproductive success is primarily constrained by the number of sperm stored when mating early in life (Fjerdingstad and Boomsma 1998), so that queens are expected to minimize sperm death after storage, rather than be inclined to kill less viable sperm, as would be selected in mating systems where females have the possibility to remate.

We found that the positive effects of seminal fluid on sperm viability were independent of the time that sperm had been stored (Fig. 3b). This reinforces the result from Fig. 3a that seminal fluid has a positive effect whenever sperm is exposed to stress factors and independent of earlier exposure to accessory gland secretion. However, it also indicates that sperm viability after 1 year of storage is essentially the same as upon ejaculation and immediately after storage because we would otherwise have expected lower scores after 1 year. Thus, although our experimental design only allows the direct comparison of relative spermenhancing effects, our data indirectly indicate that Atta queens do indeed succeed in keeping the viability of stored sperm high.

However, we did find a small difference between the curves for newly stored sperm in Fig. 3a, b (ANCOVA: year collected, $\left.F_{1}, 68=3.973, p=0.05\right)$. We have no explanation for this, but it seems most likely that this is due to methodological and/or ecological differences between years, such as the first author's improved skills in dissecting and handling Atta males and specific developmental circumstances for the ant brood, such as an earlylate start of the rainy season.

Seminal fluid function in other insects

The crucial difference between ant females (queens) with obligate multiple mating and promiscuous nonsocial insects is that ant queens obtain all their matings on a single day (i.e., during a very short period relative to their life span), whereas promiscuous nonsocial insects remate at regular intervals (Baer and Boomsma 2004; Boomsma et al. 2005; Boomsma 2007). Remating promiscuity tends to select for higher sperm viability (Hunter and Birkhead 2002; GarcíaGonzález and Simmons 2005) similar to what seems to have happened in Atta leafcutter ants, where queens mate with several males on a single day without remating later. However, the selection forces responsible are different: sperm competition seems the prime force in nonsocial insects and the need to acquire very large amounts of nonrenewable sperm is almost certainly the driving mechanism in Atta. The same trend exists in bees, where neither solitary nor eusocial species remate later in life, but where the latter species store more sperm and have higher sperm viability (Hunter and Birkhead 2002).

In spite of the fundamental differences between social insects with lifetime commitment of mating partners and promiscuous nonsocial insects (Boomsma et al. 2005), our results for leafcutter ants may help to gain new insights in noneusocial mating systems. For example, males of the Australian field cricket Teleogryllus oceanicus have been found to adjust their ejaculate quality (number of live sperm) to the risk and intensity of sperm competition (Thomas and Simmons 2007), but the mechanism to achieve this has remained unclear. Our results suggest that cricket males may alter ejaculate quality by simply changing the volume of accessory gland secretion in their spermatophores. Where much of the present literature on chemical arms races between the sexes has so far emphasized the kind and quality of hostile seminal fluid compounds, the possibility that males can actively adjust the quantity of seminal fluid in response to the likelihood of sperm competition deserves to be investigated.

Enhancing sperm viability is likely to be one of the most fundamental traits of seminal fluid. This function should 
have ancient evolutionary roots, as sperm of animals with internal fertilization always had to be transported in an ejaculate medium. Thus, even though the characteristics of social insect mating systems (no remating promiscuity, very long sperm storage) have taken sperm viabilities to high levels, many of the chemical substances responsible for maintaining viable sperm might have been conserved throughout evolutionary time. Further studies using proteomics and metabolomics techniques will have to identify whether social insects have merely optimized ancestral mixtures of accessory gland compounds or whether completely new secretions have evolved to ensure the high levels of sperm viability required.

Acknowledgements We thank Aniek Ivens and Jens Broch for collecting ants in the field, David Nash for statistical support, Francisco García-González for assistance with the Live/Dead ${ }^{\mathrm{TM}}$ protocol, and Leigh Simmons for valuable comments on the manuscript. We thank the Smithsonian Tropical Research Institute in Panama for facilities and logistic support and the Autoridad Nacional de Ambiente (ANAM) for issuing collecting and export permits. This work was supported by grants from the Danish National Research Foundation to SPAdB and JJB and an Australian Research Council Fellowship to BB.

\section{References}

Baer B (2003) Bumblebees as model organisms to study male sexual selection in social insects. Behav Ecol Sociobiol 54:521

Baer B (2005) Sexual selection in Apis bees. Apidologie 36:187-200

Baer B, Boomsma JJ (2004) Male reproductive investment and queen mating-frequency in fungus-growing ants. Behav Ecol 15:426-432

Baer B, Boomsma JJ (2006) Mating biology of the leaf-cutting ants Atta colombica and Atta cephalotes. J Morphol 267:1165-1171

Baer B, Morgan E-D, Schmid-Hempel P (2001) A nonspecific fatty acid within the bumblebee mating plug prevents females from remating. Proc Natl Acad Sci U S A 98:3926-3928

Baer B, Armitage S, Boomsma JJ (2006) Sperm storage induces an immunity cost in ants. Nature 44:872-875

Bernasconi G, Hellriegel B, Heyland A, Ward PI (2002) Sperm survival in the female reproductive tract in the fly Scathophaga stercoraria (L.). J Insect Physiol 48:197-203

Boomsma JJ (2007) Kin selection versus sexual selection: why the ends do not meet. Curr Biol 17:R673-R683

Boomsma JJ, Baer B, Heinze J (2005) The evolution of male traits in social insects. Annu Rev Entomol 50:395-420

Chapman T, Davies SJ (2004) Functions and analysis of the seminal fluid proteins of male Drosophila melanogaster fruit flies. Peptides 25:1477-1490

Chen PS (1984) The functional morphology and biochemistry of insect male accessory glands and their secretions. Annu Rev Entomol 29:233-255

Cole B (1983) Multiple mating and the evolution of social behavior in the hymenoptera. Behav Ecol Sociobiol 12:191-201

Collins AM (2000) Survival of honey bee (Hymenoptera: Apidae) spermatozoa stored at above-freezing temperatures. J Econ Entomol 93:568-571

Collins AM, Donoghue AM (1999) Viability assessment of honey bee Apis mellifera sperm using dual fluorescent staining. Theriogenology 51:1513-1523
Collins AM, Caperna TJ, Williams V, Garrett WM, Evans JD (2006) Proteomic analyses of male contributions to honey bee sperm storage and mating. Insect Mol Biol 15:541-549

Colonello NA, Hartfelder K (2005) She's my girl-male accessory gland products and their function in the reproductive biology of social bees. Apidologie 36:231-244

Damiens D, Bressac C, Brillard J-P, Chevrier C (2002) Qualitative aspects of sperm stock in males and females from Eupelmus orientalis and Dinarmus basalis (Hymenoptera: Chalcidoidea) as revealed by dual fluorescence. Physiol Entomol 27:97-102

Demary K (2005) Sperm storage and viability in Photinus fireflies. J Insect Physiol 51:837-841

Fjerdingstad EJ, Boomsma JJ (1998) Multiple mating increases the sperm stores of Atta colombica leafcutter ant queens. Behav Ecol Sociobiol 42:257-261

Fjerdingstad EJ, Boomsma JJ, Thorén P (1998) Multiple paternity in the leafcutter ant Atta colombica - a microsatellite DNA study. Heredity 80:118-126

Fry CL, Wilkinson GS (2004) Sperm survival in female stalk-eyed flies depends on seminal fluid and meiotic drive. Evolution 58:1622-1626

García-González F, Simmons LW (2005) Sperm viability matters in insect sperm competition. Curr Biol 15:271-275

Gillott C (2003) Male accessory gland secretions: modulators of female reproductive physiology and behavior. Annu Rev Entomol 48:163-184

Hodgson D, Hosken D (2006) Sperm competition promotes the exploitation of rival ejaculates. J Theor Biol 243:230-234

Hölldobler B, Bartz SH (1985) Sociobiology of reproduction in ants. In: Hölldobler B, Lindauer M (eds) Experimental behavioural ecology and sociobiology. Gustav Fischer Verlag, Stuttgart, pp 237-257

Hunter FM, Birkhead TR (2002) Sperm viability and sperm competition in insects. Curr Biol 12:121-123

Keller L (1998) Queen lifespan and colony characteristics in ants and termites. Insectes Soc 45:235-246

Koeniger N, Koeniger G (1991) An evolutionary approach to mating behaviour and drone copulatory organs in Apis. Apidologie 22:581-590

Koeniger N, Koeniger G, Wongsiri S (1989) Mating and sperm transfer in Apis florea. Apidologie 20:413-418

Kubli E (2003) Sex-peptides: seminal peptides of the Drosophila male. CMLS Cell Mol Life Sci 60:1689-1704

Mikheyev AS (2003) Evidence for mating plugs in the fire ant Solenopsis invicta. Insectes Soc 50:401-402

Poiani A (2006) Complexity of a seminal fluid: a review. Behav Ecol Sociobiol 60:289-310

Ravi Ram K, Wolfner MF (2007) Seminal influences: Drosophila Acps and the molecular interplay between males and females during reproduction. Integr Comp Biol 47:427-445

Sauter A, Brown MJF, Baer B, Schmid-Hempel P (2001) Males of social insects can prevent queens from multiple mating. Proc $\mathrm{R}$ Soc Lond B, Biol Sci 268:1449-1454

Schley P (1987) Einführung in die Technik der instrumentellen Besamung von Bienenköniginnen. Köhler Offset KG, Giessen

Simmons LW (2001) Sperm competition and its evolutionary consequences in the insects. Princeton University Press, Princeton

Thomas ML, Simmons LW (2007) Male crickets adjust the viability of their sperm in response to female mating status. Am Nat 170:190-195

Weber NA (1972) Gardening ants, the attines. The American Philosophical Society, Philadelphia

Woyke J (1983) Dynamics of entry of spermatozoa into the spermatheca of instrumentally inseminated queen honey bees. $\mathrm{J}$ Apicult Res 22:150-154

Yapici N, Kim YJ, Ribeiro C, Dickson BJ (2008) A receptor that mediates the post-mating switch in Drosophila reproductive behaviour. Nature 451:33-37 